a. Hospital de Niños Ricardo Gutiérrez, Ciudad Autónoma de Buenos Aires, Argentina.

b. Hospital de Niños Pedro de Elizalde, Ciudad Autónoma de Buenos Aires, Argentina.

c. Hospital Zonal General de Agudos Dr. Isidoro Iriarte, provincia de Buenos Aires, Argentina.

d. Hospital Pediátrico Dr. Avelino Lorenzo Castelán, Resistencia, Argentina.

e. Hospital de Niños Víctor J. Vilela, Rosario, Argentina.

f. Fundación Hospitalaria, Ciudad Autónoma de Buenos Aires, Argentina.

g. Hospital General de Agudos Bernardino Rivadavia, Ciudad Autónoma de Buenos Autónoma de Buenos,
Aires, Argentina.

h. Hospital Nacional Profesor Alejandro Posadas, provincia de Buenos Aires, Argentina.

i. Hospital General de Agudos Dr. Juan Antonio Fernández, Ciudad Autónoma de Buenos Aires, Argentina.

j. Hospital Pediátrico Alexander Fleming y Hospital Dr. Ramón Carrillo, Mendoza, Argentina.

k. Hospital de Niños De la Santísima Trinidad, Córdoba Argentina.

Córdoba, Arg

Hospital de Niños Dr. Humberto Notti, Mendoza, Argentina.

m. Hospital Regional Ushuaia Gobernador Ernesto Campos, Ushuaia, Argentina.

Argentina.
Hospital Municipal Materno Infantil de San Isidro, provincia de Buenos Aires, Argentina.

Hospital Luisa C. de Gandulfo y Hospital Gandulfo y Hospital Dr. Arturo Oñativia,
provincia de Buenos Aires, Argentina.

p. Hospital de Clínicas José de San Martin, Ciudad Autónoma de Buenos Autónoma de Buen, Argentina.

q. Sanatorio Franchin, Ciudad Autónoma de Buenos Aires, Argentina.

Red de Pediatría COVID-19.

Correspondencia:

Ángela Gentile: angelagentile21@gmail. com

Financiamiento: Esta investigación se realizó en el marco de una beca de investigación otorgada y financiada por la Sociedad Argentina de Pediatría.

Conflicto de intereses: Ninguno que declarar.

Recibido: $14-6-2021$ Aceptado: 8-7-2021

\section{Estudio multicéntrico de casos confirmados de COVID-19: datos preliminares de 2690 pacientes pediátricos en Argentina durante el primer año de la pandemia}

A multicenter study of confirmed COVID-19 cases:

preliminary data on 2690 pediatric patients in Argentina

during the first year of the pandemic

Ángela Gentile ${ }^{a}$ (D), María del Valle Juárez ${ }^{a}$ (D), Lucía Romero Bollón ${ }^{a}$ (D),

Aldo D. Cancellara ${ }^{(\mathbb{D}}$, Marina Pasinovich ${ }^{b}$ (D), Martín Brizuela ${ }^{c}$ (D),

Cristina Euliarted (D), Gabriela N. Ensinck $^{(\mathbb{D})}$, Carlota Russ $^{(\mathbb{D})}$,

Liliana Saracenis (D), Gabriela Tapponier ${ }^{h}$ (D), Susana Villa Nova ${ }^{i}$ (D),

Andrea Falaschi $\mathbb{D}^{\mathbb{D}}$, Analía Garnero ${ }^{k}$ (D), Pablo Melonaril $\mathbb{D}$,

Luciana Bellone $^{m}$ (D), Alejandra Gaiano ${ }^{n}$ (D) , Victor Pebe Florian $^{\circ}$ (D),

Elizabeth Bogdanowicz ${ }^{p}(\mathbb{D})$ M. Soledad Areso ${ }^{(\mathbb{D})}$, Red de Pediatría COVID-19r

\section{RESUMEN}

Introducción. La evidencia actual indica que la gravedad de la enfermedad por el coronavirus 2019 (COVID-19, por su sigla en inglés) es menor en la población pediátrica, los datos locales aún son limitados. Objetivo: caracterizar los aspectos clínicos y epidemiológicos de la infección por COVID-19 en menores de 18 años en Argentina. Población y métodos. Estudio transversal, observacional y analítico de casos confirmados de COVID-19 entre 0 y 18 años asistidos entre marzo de 2020 y marzo de 2021 en 19 centros pediátricos de referencia de Argentina. Se realizó un análisis multivariado para identificar las variables predictoras de cuadros graves.

Resultados. Se incluyeron 2690 casos de COVID-19: $77,7 \%$ residentes del área metropolitana de Buenos Aires, 50,1 \% de sexo masculino, mediana de edad de 5,6 años. El $90 \%$ ocurrió entre las semanas epidemiológicas 2047 del 2020; 60,4\% con antecedente de contacto con personas con COVID-19; y 96,6 \% en el entorno familiar. El 51,4 \% presentó síntomas respiratorios; $61,6 \%$ síntomas generales; $18,8 \%$ síntomas gastrointestinales; $17,1 \%$ síntomas neurológicos; $7,2 \%$ otros y $21,5 \%$ fueron asintomáticos. El 59,4\% fue hospitalizado; 7,4\% fueron graves o críticos. Se registraron 57 casos antecedente de asma, displasia broncopulmonar, cardiopatía congénita, desnutrición moderada a grave, obesidad, enfermedad neurológica crónica y/o edad menor de 6 meses resultaron predictores independientes de gravedad. Residir en barrios vulnerables resultó protector.

Conclusiones. Más de la mitad de los casos refirieron antecedente de contacto con personas con COVID-19 en el entorno familiar. La hospitalización no respondió a criterios clínicos de gravedad. La gravedad se encuentra asociada a la existencia de ciertas comorbilidades.

Palabras clave: infecciones por coronavirus, COVID-19, niño, sindrome inflamatorio multisistémico en niños.

http:/ / dx.doi.org/10.5546/ aap.2022.80

Texto completo en inglés:

http: / / dx.doi.org/10.5546/ aap.2022.eng.80

Cómo citar: Gentile Á, Juárez MV, Romero Bollón L Cancellara AD, et al. Estudio multicéntrico de casos confirmados de COVID-19: datos preliminares de 2690 pacientes pediátricos en Argentina durante el primer año de la pandemia. Arch Argent Pediatr 2022;120(2):80-88. de síndrome inflamatorio multisistémico. El

Red de Pediatría COVID-19:

Dra. Ma. Florencia Lucion ${ }^{a}$, Dra. Claudia Ferrario ${ }^{b}$, Dra. Norma Schenone,

Dra. Verónica Kozickic, Dra. Evangelina Zubimendie, Dra. María de los Ángeles Ausburye,

Dr. Daniel Giansiracusaf, Dra. M. Mercedes Gilf, Dra. M. Agustina Chaplin',

Dra. Silvia Pedemontes, Dra. Carola Bayle ${ }^{h}$, Dra. Valeria Nivela ${ }^{h}$,

Dra. Graciela Suárezi, Dra. Miyuki Takata ${ }^{i}$ Lic. Lorena Galasso', Dr. Leonardo Manino',

Dra. Eugenia Martínezi, Dra. Karina Rodríguezi, Dra. M. Shirley Gareis ${ }^{k}$,

Dr. Andrés Gomilak, Dra. Liliana Limal, Dra. Verónica Del Negrol,

Dra. Constanza Lovrics ${ }^{\circ}$, Dra. Leila Menta ${ }^{\circ}$ Dra. Belén Hunter ${ }^{\circ}$, Dra. Karina Blanco ${ }^{\circ}$,

Dr. Diego Ripeau ${ }^{p}$ Dr. Néstor Abramovich ${ }^{p}$ y Dra. M. Laura Verdier ${ }^{q}$ 
Registro de la investigación: PRIISA.BA Estudio multicéntrico de casos confirmados de COVID 19 en pacientes pediátricos en Argentina. $\mathrm{N}^{\circ}$ de Registro: 1835. Fecha de inscripción: 23 de junio de 2020.

\section{INTRODUCCIÓN}

Desde la declaración de la pandemia mundial el pasado 11 de marzo de 2020, la enfermedad causada por el coronavirus de tipo 2 del síndrome respiratorio agudo grave (SARS-CoV-2), conocida como COVID-19 (por su sigla en inglés), se ha diseminado por todo el mundo con un enorme impacto, no solo en el ámbito sanitario sino también en el ámbito económico y social. ${ }^{1}$ En Argentina, el primer caso se registró el 3 de marzo de 2020 y, para abril de 2021, se habían notificado más de 136 millones de casos confirmados y 2,9 millones de defunciones en todo el mundo. ${ }^{2}$

Datos iniciales señalaron que la población infantil y adolescente es susceptible de infectarse, con mejor pronóstico en comparación con los adultos. ${ }^{3,4}$ En general, tienen síntomas leves y suelen recuperarse al cabo de 1 a 2 semanas desde del inicio de la enfermedad; el $15-35 \%$ puede ser asintomático. ${ }^{5}$

En Argentina, hasta la semana epidemiológica (SE) 11 de 2021, durante la "primera ola" de la pandemia, el 9,4\% de los casos se registraron en menores de 20 años con una letalidad $0,08 \%{ }^{6}$ La vigilancia epidemiológica de COVID-19 en la población pediátrica tuvo características particulares desde su inicio, producto de la limitada información con la que se contaba y la mayor afectación en los adultos. ${ }^{7}$

En mayo de 2020, se reportó la existencia de un síndrome multisistémico en pacientes pediátricos asociado a la infección por el SARS-CoV-2, que en muchos casos evolucionaban hacia la falla multiorgánica. ${ }^{8-10}$ La información local sobre las características de la COVID-19 en la población pediátrica sigue siendo limitada. ${ }^{11,12}$

Los objetivos de la presente investigación son caracterizar la infección por COVID-19 en niños menores de 18 años en Argentina según aspectos clínicos y epidemiológicos, evaluar las características clínicas de los pacientes internados con infección confirmada e identificar factores de riesgo de gravedad.

\section{POBLACIÓN Y MÉTODOS}

Estudio transversal, multicéntrico, observacional y analítico. Se incluyeron todos los pacientes menores de 18 años con infección confirmada por SARS-CoV-2 (según la definición del Ministerio de Salud de la Nación) ${ }^{7}$ asistidos en los centros participantes en forma retrospectiva (1 de marzo al 30 de septiembre de 2020) y prospectiva (1 de octubre al 31 de marzo 2021). Participaron 19 centros ubicados en Ciudad Autónoma de Buenos Aires (CABA) y las provincias de Buenos Aires, Chaco, Córdoba, Mendoza, Santa Fe y Tierra del Fuego.

\section{Recolección de los datos}

Los datos fueron obtenidos por los investigadores de los centros participantes. Se diseñó una ficha específica para la recopilación de los datos epidemiológicos y clínicos. Se registraron datos sobre: el centro participante, el código de identificación del paciente, la fecha de ingreso, los datos demográficos, los datos de hospitalización (casos ingresados a sala y diagnóstico al ingreso), el tiempo de evolución de los síntomas, el contacto con caso confirmado de COVID-19 y / o infección respiratoria aguda alta (IRA), la presencia de comorbilidades (prematuridad, enfermedad respiratoria crónica o recidivante, inmunosupresión, desnutrición, cardiopatía congénita, enfermedad neurológica, hepática, renal y metabólica), signos y síntomas, estudios complementarios por imágenes y de laboratorio (si se hubiesen indicado por parte del médico tratante), la evolución clínica y el tratamiento recibido.

Se consideró hacinamiento crítico de acuerdo con la definición del Instituto Nacional de Estadísticas y Censos (INDEC): convivencia de más de tres personas por cuarto (sin considerar la cocina y el baño); y se consideraron barrios vulnerables o populares, según el Registro Nacional de Barrios Populares, aquellos en donde viven al menos ocho familias agrupadas o contiguas, con más de la mitad de la población sin título de propiedad del suelo ni acceso regular a dos o más de los servicios básicos.

La gravedad de la COVID-19 se definió sobre la base de las características clínico-radiológicas según Dong y colaboradores. ${ }^{13}$ La coordinación del estudio, la carga de datos y el análisis se realizó en la División de Promoción y Protección de la Salud del Hospital de Niños Ricardo Gutiérrez de la Ciudad Autónoma de Buenos Aires.

\section{Diagnóstico etiológico}

Los casos fueron confirmados mediante el método de reacción en cadena de la polimerasa con transcriptasa reversa (RT-PCR, 
por su sigla en inglés) para SARS-CoV-2 en aspirado nasofaríngeo o hisopado de fauces. Se aceptaron pruebas diagnósticas adicionales a la inmunoflorescencia indirecta (IFI), como film array y PCR ampliada, en la búsqueda de otros agentes etiológicos virales responsables de las IRAG: virus sincicial respiratorio (VSR), adenovirus (AV), influenza (IF) A y B, y parainfluenza (PIF) 1, 2 y 3.

En aquellos pacientes con diagnóstico de síndrome inflamatorio multisistémico posCOVID-19 (SIM-C) se aceptó el resultado de serología positiva para SARS-CoV-2 como confirmatorio, según las normativas del Ministerio de Salud.

\section{Análisis estadístico}

Se realizó una descripción general calculando la media y la desviación estándar o la mediana y el rango intercuartílico (RIC) para las variables numéricas según su distribución. Para las variables categóricas, se utilizaron proporciones y sus intervalos de confianza del $95 \%$ (IC95\%). Para el análisis de las variables continuas se utilizó la prueba t o prueba de Wilcoxon. Para las variables categóricas, se utilizaron la prueba de chi cuadrado con la corrección de Yates o la prueba de Fisher.

Para el análisis de los resultados de exámenes complementarios, se excluyeron los casos con diagnóstico de SIM-C. Se construyó un modelo de regresión logística múltiple para identificar las variables predictoras de enfermedad grave. Se consideraron como casos graves los clasificados como graves y críticos según la clasificación de
Dong, ${ }^{13}$ y como casos no graves, los asintomáticos, leves o moderados. Se utilizó el programa STATA/SE versión $13^{a}$ para el análisis estadístico. La medida de asociación utilizada fue la razón de momios (OR, por su sigla en inglés) con un IC95 \%. En el modelo final se evaluaron la calibración y la discriminación, la primera mediante la prueba de Hosmer y Lemeshow en deciles de riesgo, y la segunda mediante el área bajo la curva con características del receptoroperador (ROC, por su sigla en inglés). Se consideraron adecuadas una calibración global con una $p>0,05$ y una discriminación con un área bajo la curva ROC $>0,7$.

\section{Consideraciones éticas}

Se codificaron todas las fichas antes de su análisis para asegurar el anonimato, de acuerdo con la Declaración de Helsinki y de la ley de Habeas Data (Ley N. ${ }^{\circ}$ 25326). Esta investigación fue evaluada y aprobada por los comités de docencia y de ética en investigación de los centros participantes.

\section{RESULTADOS}

Se incluyeron, entre la SE 12 de 2020 y la SE 11 de 2021, un total de 2690 casos confirmados de COVID-19. E1 77,7 \% residían en el área metropolitana de Buenos Aires (AMBA) -CABA $(40,5 \%)$ y provincia de Buenos Aires $(37,2 \%)-$ seguido por Chaco $(6,5 \%)$, Mendoza $(6,4 \%)$, Santa Fe $(5,3 \%)$, Córdoba $(2,5 \%)$, Tierra del Fuego (1,4\%), Chubut (0,1\%) y Misiones $(0,1 \%)$ (Figura 1). El $90 \%$ de los casos ocurrieron entre las

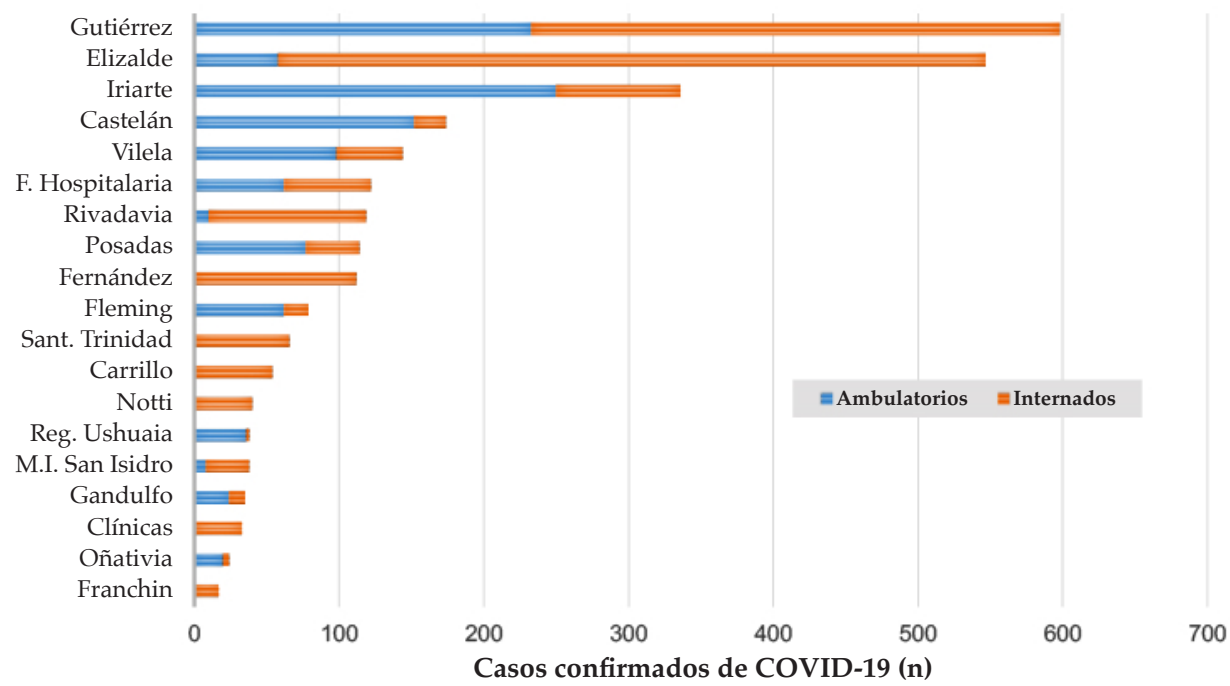


SE 20 y 47 (10 de mayo a 21 de noviembre), con un pico en la SE 32 (agosto) (Figura 2).

\section{Características de los casos confirmados}

Entre los casos confirmados $(\mathrm{n}=2690)$, la mediana de edad fue de 5,6 años (RIC: 1,311,3 años); el 21,2 \% eran menores de 1 año, con una distribución equitativa por sexos. El 29,9\% de los casos vivía en barrios populares, y 28,7 \% en condiciones de hacinamiento crítico. El 60,4\% tenía antecedente de contacto con personas con infecciones respiratorias agudas y / o COVID-19 confirmada por laboratorio, sin diferencias entre los grupos etarios. El 96,6\% refirió que el contacto

Figura 2. Distribución de los casos de COVID-19 según la semana epidemiológica $(n=2690)$

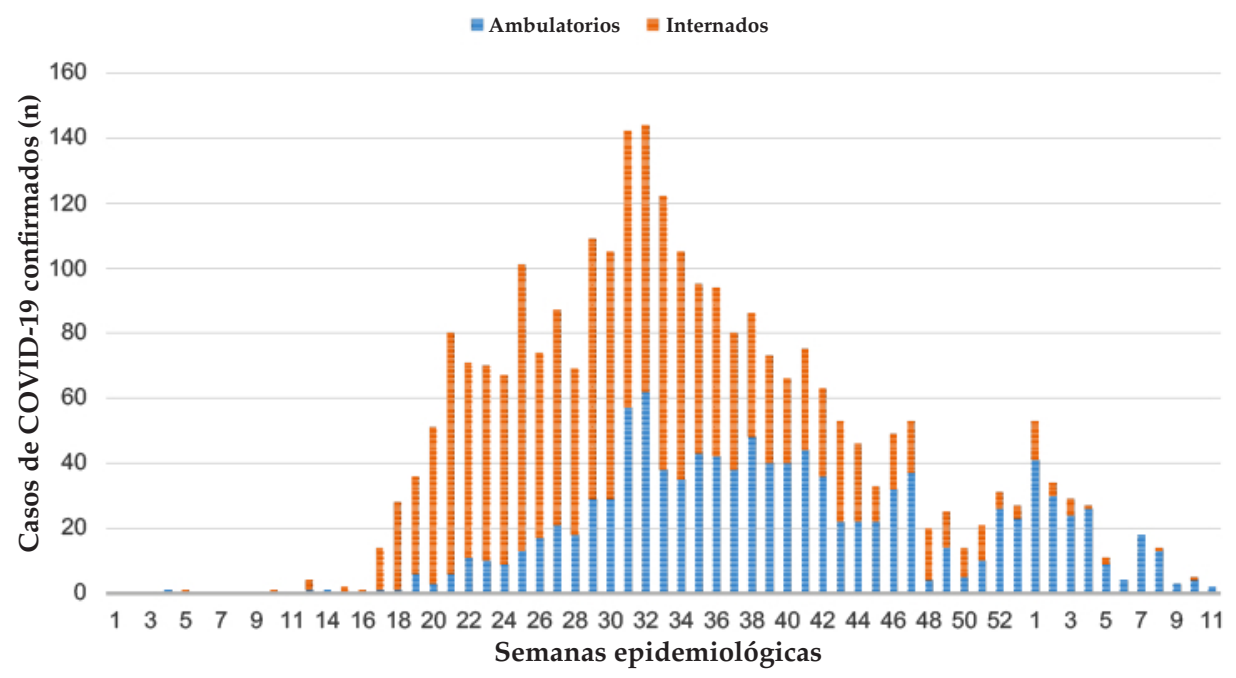

FIgURA 3. Perfil de presentación clínica de los casos confirmados de COVID-19, según grupos etarios $(n=2690)$

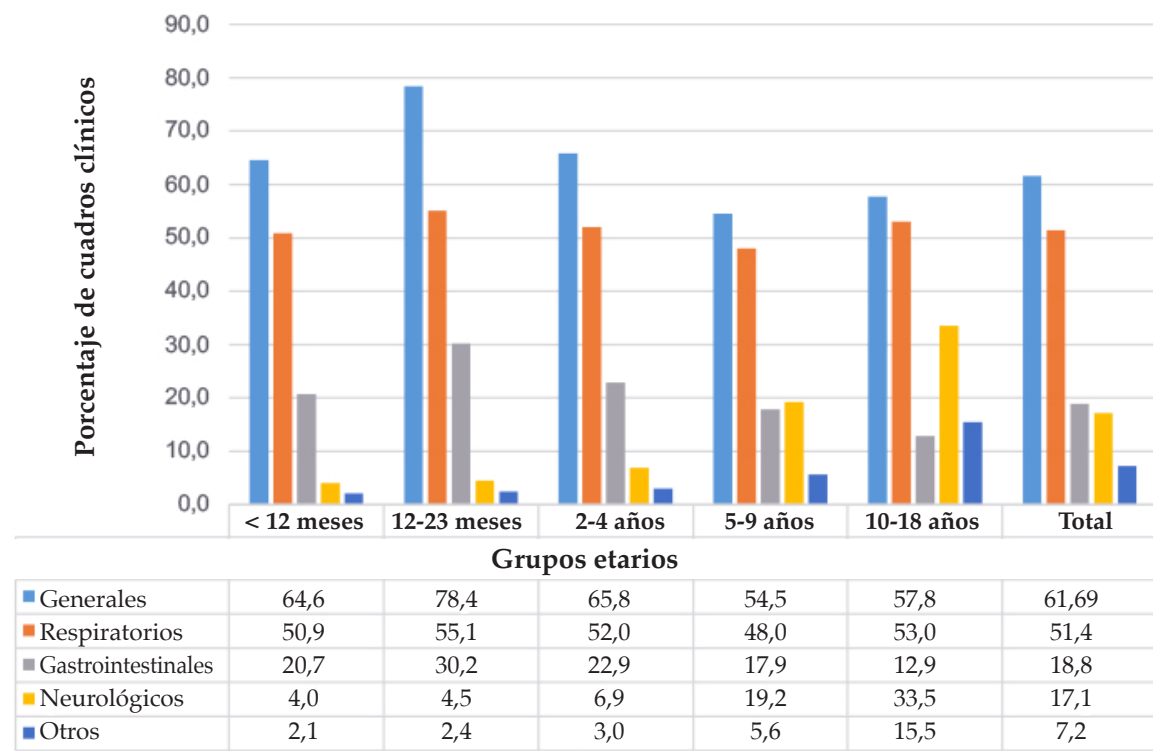

Nota: algunos casos tienen más de un signo o síntoma de presentación.

Referencias de los síntomas:

- Generales: fiebre, malestar y mialgias.

- Respiratorios: tos, odinofagia, rinorrea y dificultad respiratoria.

- Gastrointestinales: vómitos, náuseas, diarrea y dolor abdominal.

- Neurológicos: cefalea y convulsiones.

- Otros: anosmia, disgeusia y exantema inespecífico. 
con caso confirmado de COVID-19 había ocurrido en el entorno familiar.

El 59,4\% $(\mathrm{n}=1599)$ fue hospitalizado ( $\mathrm{RIC}=28,5-98,5 \%)$, con una distribución heterogénea en los distintos períodos y jurisdicciones. Las tasas de hospitalización resultaron estadísticamente diferentes; los niños menores de 1 año tuvieron la mayor tasa de hospitalización (29,6\%). El 23,4 \% de los casos tenían una o más comorbilidades y la enfermedad respiratoria fue la más frecuente $(52,5 \%)$. En la Tabla 1 se describen las características clínicoepidemiológicas de la población comparando los casos ambulatorios y hospitalizados.

El 21,5\% fueron asintomáticos. Los signos y síntomas más frecuentes fueron la fiebre $>37,5^{\circ} \mathrm{C}(69,4 \%)$, tos $(35,9 \%)$, rinorrea $(20,7 \%)$ y odinofagia $(20,5 \%)$. En la Figura 3 se muestran

TABla 1. Características clínico-epidemiológicas y frecuencia de comorbilidades de los casos confirmados de COVID-19 (total, ambulatorios y hospitalizados; $n=2690$ )

\begin{tabular}{|c|c|c|c|c|}
\hline Características de la población & $\begin{array}{c}\text { Total } \\
(\mathrm{n}=2690) \\
\%(\mathrm{n})\end{array}$ & $\begin{array}{c}\text { Ambulatorios } \\
(\mathbf{n}=1091) \\
\%(\mathbf{n})\end{array}$ & $\begin{array}{c}\text { Hospitalizados } \\
(\mathbf{n}=\mathbf{1 5 9 9 )} \\
\%(\mathbf{n})\end{array}$ & $p$ \\
\hline Edad en años (mediana; rango intercuartílico) & $5,6(1-11)$ & $7,6(3-13)$ & $4,1(1-10)$ & $<0,001$ \\
\hline $\begin{array}{l}\text { Grupos etarios: } \\
\quad<12 \text { meses } \\
12-23 \text { meses } \\
2-4 \text { años } \\
\text { 5-9 años } \\
\geq 10 \text { años }\end{array}$ & $\begin{array}{l}21,2 \%(570) \\
9,1 \%(245) \\
16,1 \%(433) \\
22,5 \%(604) \\
31,1 \%(838)\end{array}$ & $\begin{array}{l}8,8 \%(96) \\
11,4 \%(124) \\
16,9 \%(185) \\
25,0 \%(273) \\
37,9 \%(413)\end{array}$ & $\begin{array}{l}29,6 \%(474) \\
7,6 \%(121) \\
15,5 \%(248) \\
20,7 \%(331) \\
26,6 \%(425)\end{array}$ & $<0,001$ \\
\hline Sexo masculino & $50,1 \%(1349)$ & $50,3 \%(549)$ & $50,0 \%(800)$ & 0,913 \\
\hline $\begin{array}{l}\text { Semana epidemiológica de inicio de síntomas } \\
\text { (semana, rango de semanas) }\end{array}$ & $32(25-38)$ & $35(27-41)$ & $30(24-36)$ & $<0,001$ \\
\hline Residencia en AMBA & $77,7 \%(2092)$ & $68 \%(742)$ & $84,4 \%(1350)$ & $<0,001$ \\
\hline Residencia en barrio popular & $29,9 \%(805)$ & $19,2 \%(210)$ & $37,2 \%(595)$ & $<0,001$ \\
\hline Hacinamiento crítico & $28,7 \%(774)$ & $12,1 \%(132)$ & $40,1 \%(642)$ & $<0,001$ \\
\hline Contacto con persona con COVID-19 & $60,3 \%(1623)$ & $50,2 \%(548)$ & $67,2 \%(1075)$ & $<0,001$ \\
\hline Contacto con persona con IRA & $24,3 \%(654)$ & $17,6 \%(192)$ & $28,9 \%(462)$ & $<0,001$ \\
\hline $\begin{array}{l}\text { Contacto con persona con COVID-19 } \\
\text { en el entorno familiar }\end{array}$ & $96,6 \%(1543)$ & $97,9 \%(527)$ & $95,4 \%(1016)$ & 0,049 \\
\hline $\begin{array}{l}\text { Comorbilidades } \\
\text { Enfermedad respiratoria } \\
\qquad \begin{array}{l}\text { Asma } \\
\text { Sibilancias recurrentes } \\
\text { Displasia broncopulmonar } \\
\text { Dificultad respiratoria perinatal } \\
\text { Fibrosis quística }\end{array}\end{array}$ & $\begin{array}{l}23,4 \%(630) \\
(52,5 \%) \\
6,5 \%(174) \\
4,8 \%(130) \\
0,6 \%(18) \\
0,2 \%(6) \\
0,1 \%(3)\end{array}$ & $\begin{array}{l}12,1 \%(131) \\
5,3 \%(58) \\
3,1 \%(34) \\
0,1 \%(1) \\
0 \%(0) \\
0,1 \%(1)\end{array}$ & $\begin{array}{l}7,2 \%(116) \\
6 \%(96) \\
1,1 \%(17) \\
0,4 \%(6) \\
0,1 \%(2)\end{array}$ & $\begin{array}{c}<0,001 \\
0,053 \\
<0,001 \\
0,001 \\
0,087 \\
1\end{array}$ \\
\hline $\begin{array}{l}\text { Enfermedad metabólica } \\
\text { Obesidad } \\
\text { Diabetes }\end{array}$ & $\begin{array}{c}(8,4 \%) \\
1,3 \%(36) \\
0,6 \%(17)\end{array}$ & $\begin{array}{l}0,2 \%(2) \\
0,5 \%(5)\end{array}$ & $\begin{array}{l}2,1 \%(34) \\
0,7 \%(12)\end{array}$ & $\begin{array}{c}<0,001 \\
0,489\end{array}$ \\
\hline $\begin{array}{l}\text { Inmunodeficiencia } \\
\qquad \begin{array}{l}\text { Primaria } \\
\text { Enfermedad oncohematológica } \\
\text { Inmunodeficiencia adquirida } \\
\text { Trasplante } \\
\text { Tratamiento inmunosupresor }\end{array}\end{array}$ & $\begin{array}{l}(3,2 \%) \\
0,6 \%(15) \\
1,5 \%(40) \\
0.2 \%(6) \\
0,04 \%(1) \\
0,9 \%(25)\end{array}$ & $\begin{array}{l}0,2 \%(2) \\
0,6 \%(7) \\
0 \%(0) \\
0 \%(0) \\
0,4 \%(4)\end{array}$ & $\begin{array}{l}0,81 \%(13) \\
2,1 \%(33) \\
0.4 \%(6) \\
0,06 \%(1) \\
1,3 \%(21)\end{array}$ & $\begin{array}{c}0,034 \\
0,004 \\
0.087 \\
1 \\
0,012\end{array}$ \\
\hline Desnutrición & $0,7 \%(18)$ & $0,1 \%(1)$ & $1,1 \%(17)$ & 0,001 \\
\hline Enfermedad neurológica crónica & $3,3 \%(88)$ & $0,8 \%(9)$ & $4,9 \%(79)$ & $<0,001$ \\
\hline Cardiopatía congénita & $1,3 \%(34)$ & $0,2 \%(2)$ & $2 \%(32)$ & $<0,001$ \\
\hline Enfermedad renal crónica & $1,1 \%(29)$ & $0,3 \%(3)$ & $1,6 \%(26)$ & $<0,001$ \\
\hline Enfermedad hepática & $0,3 \%(9)$ & $0,2 \%(2)$ & $0,4 \%(7)$ & 0,326 \\
\hline Prematuridad & $1,7 \%(45)$ & $0,4 \%(4)$ & $2,6 \%(41)$ & $<0,001$ \\
\hline
\end{tabular}

AMBA: Área Metropolitana de Buenos Aires, IRA: infección respiratoria aguda, COVID-19: enfermedad por el nuevo coronavirus 2019. 
los signos y síntomas agrupados y por grupo etario; los niños de 10 años o más presentaron un porcentaje significativamente mayor de síntomas neurológicos (cefalea) y anosmia con o sin disgeusia.

El 5,6 \% se presentaron inicialmente como infección respiratoria aguda baja (bronquiolitis $2,5 \%$ y neumonía $3,1 \%$ ) y el 3,6 \% como SIM-C.

\section{Evolución y gravedad de los casos confirmados hospitalizados}

Entre los casos confirmados hospitalizados $(\mathrm{n}=1599)$, la mediana de tiempo de internación fue de 7 días (RIC: 3-9 días). Se registraron 57 pacientes con diagnóstico de SIM-C, con una mediana de edad de 6,5 años. En este grupo, el 61,4\% recibió gammaglobulina; el 59,6\% recibió corticoides; y el 52,6\% requirió cuidados intensivos.

Para el análisis de los estudios complementarios y la evolución, se excluyeron los SIM-C, debido a las características diferenciales de este síndrome.

Entre los 1542 casos hospitalizados (excluidos los SIM-C), se solicitó radiografía de tórax al $30,9 \%(68,1 \%$ con resultado normal; $14,6 \%$ con infiltrado intersticial; 7,3\% con condensación y $4,6 \%$ con atrapamiento aéreo) y tomografía axial computada de tórax al 1,3\% (10 eran normales, 6 presentaban imagen en vidrio esmerilado, 2 tenían derrame pleural y, el resto, otros infiltrados). Se buscaron otros virus respiratorios en el 2,6\% de los casos $(n=41)$ y se detectó coinfección viral en 5 casos con adenovirus, metapneumovirus y rinovirus.

En el 52,1\% $(n=804)$ se realizó laboratorio al ingreso; los pacientes con cuadros graves al inicio presentaban mayores valores de leucocitos, neutrófilos, eritrosedimentación, proteína $C$ reactiva, lactato deshidrogenasa, urea y ferritina y disminución del tiempo de protrombina, con una diferencia estadísticamente significativa respecto a los pacientes no graves.

En la Figura 4 se muestra la distribución por grupos etarios según la clasificación final. El 7,4 \% $(\mathrm{n}=114)$ se clasificaron como graves o críticos: el $84,2 \%$ requirió oxígeno; el $24,5 \%$ cuidados intensivos, y el 17,5\% asistencia respiratoria mecánica. El análisis multivariado (Tabla 2) determinó como predictores independientes de gravedad de COVID-19 el antecedente de asma, displasia broncopulmonar, cardiopatías congénitas, desnutrición moderada a grave, obesidad, enfermedades neurológicas crónicas y edad menor de 6 meses; mientras que el antecedente de residir en barrio vulnerable fue un predictor independiente, pero con efecto protector.

Se registraron 8 fallecimientos (mediana de edad de 8,5 años; rango = 0-16 años), todos presentaban comorbilidades: enfermedad renal crónica (2), cardiopatía (2) y los restantes, enfermedad pulmonar crónica, inmunodeficiencia primaria, enfermedad hepática crónica y debut oncohematológico.

FIGURA 4. Distribución porcentual de casos confirmados de COVID-19 hospitalizados según su clasificación final al egreso por grupos etarios $(n=1542)$

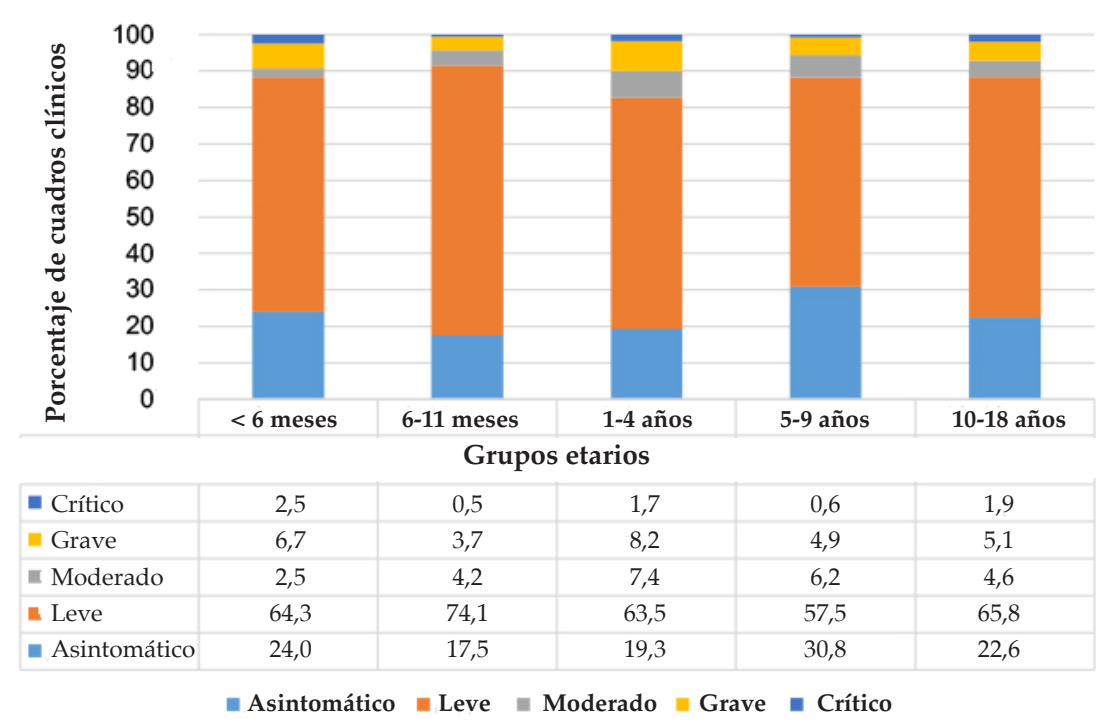




\section{DISCUSIÓN}

Para entender las estrategias de manejo clínico y epidemiológico en pediatría es fundamental explicitar las características diferenciales de la infección por el SARS-CoV-2 en este grupo etario. ${ }^{14}$ En el presente estudio se muestran las características clínicas y epidemiológicas de los pacientes con diagnóstico confirmado de COVID-19 atendidos en 19 centros pediátricos de referencia en Argentina. El pico epidémico se observó diez semanas antes de lo descripto a nivel nacional, probablemente debido a que la mayoría de los casos fueron incluidos por hospitales del AMBA, primera región afectada en el país desde el punto de vista cronológico. ${ }^{15}$

Las diferencias observadas en los porcentajes de internación entre los centros reflejan la dinámica de la estrategia de manejo de casos en pediatría en el país. ${ }^{16}$ Inicialmente, se indicaba la internación de todos los pacientes pediátricos (aún las formas clínicas leves), después a los menores de un año y, por último, la edad por debajo de los 6 meses fue criterio de internación, independientemente de la gravedad. Esta estrategia tuvo como objetivo principal asegurar el aislamiento del paciente con sus cuidadores y fue determinante la información limitada que existía sobre la enfermedad en pediatría, según la cual los niños eran considerados "hipercontagiadores", tomando como referencia las características epidemiológicas de la gripe. ${ }^{17} \mathrm{De}$ acuerdo a estas consideraciones, se puede afirmar que la internación pediátrica por COVID-19 en el año 2020 no respondió necesariamente a un criterio de gravedad de la enfermedad, como sílo fue en adultos. ${ }^{18,19}$ En este punto, se señala como limitación de este estudio que algunos centros solo incluyeron los casos hospitalizados y que los criterios epidemiológicos de internación difirieron entre las jurisdicciones.

En esta serie, en términos generales, los niños presentaban contacto previo con casos de COVID-19 confirmados en el entorno familiar, evidencia que, en porcentajes variables, también se encuentra en la bibliografía. ${ }^{15,20} \mathrm{Un}$ tercio de ellos vivían en barrios populares con hacinamiento crítico, dado que la trasmisión de SARS-CoV-2 es por contacto y gota respiratoria, condiciones que favorecen la transmisión. En CABA, un estudio de prevalencia realizado en dichos barrios demostró una alta tasa de infecciones no detectadas (probablemente asintomáticas). ${ }^{21}$

La mediana de edad fue de 5 años, similar a lo descripto en todo el mundo. ${ }^{22} \mathrm{Al}$ inicio de la pandemia, en un marco de acceso limitado a las pruebas diagnósticas y tomando como referencia que la enfermedad en adultos tenía características semejantes a la gripe, se asumió como una vigilancia de virus respiratorios, considerando como obligatoria la presencia de fiebre y los signos y síntomas respiratorios para la definición de caso sospechoso.

Uno de cada cinco niños fue asintomático, lo cual destaca la importancia de la detección a través de las pruebas de diagnóstico molecular en pediatría, con alta sensibilidad y especificidad, limitando el uso de pruebas de antígeno rápidas a los casos sintomáticos. ${ }^{23}$ Se realizó la búsqueda de otros virus en solo el $2 \%$ de los casos: un

TABLA 2. Análisis multivariado de factores asociados a presentación grave de COVID-19 en pacientes hospitalizados ( $n=1542)$

\begin{tabular}{lccc}
\hline Variable & OR & IC95\% & $p$ \\
\hline Asma & 5,7 & $3,3-9,7$ & $<0,001$ \\
Displasia broncopulmonar & 6,3 & $2,0-20,0$ & 0,001 \\
Obesidad & 3,7 & $1,5-9,2$ & 0,005 \\
Cardiopatía & 4,6 & $1,9-11,6$ & $<0,001$ \\
Enfermedad neurológica & 2,2 & $1,1-4,7$ & 0,032 \\
Desnutrición & 5,1 & $1,6-16,2$ & 0,006 \\
Residencia en barrio vulnerable & 0,4 & $0,2-0,7$ & $<0,001$ \\
Prematuridad & 3,1 & $0,3-30,6$ & 0,318 \\
Diabetes & 1,9 & $0,4-9,9$ & 0,445 \\
Menor de 6 meses & 1,9 & $1,1-3,1$ & 0,009 \\
Enfermedad renal crónica & 1,5 & $0,4-5,4$ & 0,549 \\
Enfermedad oncohematológica & 2,3 & $0,7-7,1$ & 0,127 \\
\hline
\end{tabular}

Nota: el modelo mostró una calibración adecuada cuando fue evaluado con la prueba de Hosmer-Lemeshow $(\mathrm{p}=0,328)$ y discriminó de manera aceptable, evaluado mediante la curva ROC, presentando un área bajo la curva de 0,729 (IC95 \%: 0,68- 0,72).

OR: razón de momios (por su sigla en inglés), IC95\%: intervalo de confianza del $95 \%$. 
desafío actual será evaluar el comportamiento de los virus estacionales en pediatría fuera del confinamiento. ${ }^{24}$

Dada la presentación asintomática u oligosintomática en pediatría, se destaca la importancia de sensibilizar la definición de caso, jerarquizar el antecedente epidemiológico de contacto estrecho y considerar las posibilidades de un contagio a partir de un adulto para cortar la cadena de transmisión. ${ }^{25}$

En cuanto a los tipos de presentación clínica, a partir de los 10 años se observa el mayor porcentaje de anosmia, disgeusia y cefalea semejante al cuadro que presentan los adultos. ${ }^{26,27}$ El $67 \%$ de los casos fueron asintomáticos o presentaron un cuadro clínico leve, lo cual coincide con lo publicado hasta la fecha en pediatría. ${ }^{28,29}$ Por otra parte, dada la menor gravedad, los estudios por imágenes complementarios no se solicitaron por protocolo, sino de acuerdo a los estándares y disponibilidad de cada centro participante, y en su mayoría resultaron normales. ${ }^{30}$

El $7 \%$ presentó un cuadro grave de COVID-19, con una letalidad de 0,3\% (todos con comorbilidades), mortalidad comparable con lo descripto a nivel nacional e internacional. ${ }^{31,32}$ Las comorbilidades que se relacionaron significativamente con la evolución a cuadros graves fueron el asma, la displasia broncopulmonar, la obesidad, las enfermedades neurológicas crónicas, las cardiopatías y la desnutrición, así como la edad menor a 6 meses. ${ }^{33,34} \mathrm{El}$ antecedente de residencia en barrios vulnerables se comportó como un factor de protección; ante la necesidad de garantizar el aislamiento, este grupo poblacional se hospitalizó, en su mayoría, independientemente de la edad o presencia de comorbilidades, hasta que estuvieron disponibles los dispositivos de aislamiento extrahospitalarios de acuerdo a la normativa nacional. ${ }^{16}$

Los pacientes con cuadros graves presentaron al inicio mayores valores de leucocitos y neutrófilos, lo cual se diferencia de lo evidenciado en adultos, ya que existen numerosas publicaciones que asocian la presencia de linfopenia al inicio del cuadro con una evolución más desfavorable. ${ }^{35}$ Por el contrario, el antecedente de residencia en barrios populares se comportó como un fuerte factor de protección, esto es consecuente con la evidencia que señala que la letalidad por COVID-19 fue inferior en estos barrios respecto a la población general. ${ }^{36}$
Uno de los desafíos en pediatría será evaluar también las características particulares de los casos de SIM-C y las secuelas a largo plazo que podría dejar esta enfermedad. ${ }^{37}$ Eso será objeto de otra publicación por parte de este grupo.

En Argentina, al igual que en otras partes del mundo, resultó difícil la caracterización de COVID-19 en pediatría. Además, los largos períodos de confinamiento no permitieron detectar otras enfermedades y fueron motivo de consultas tardías y disminución de controles pediátricos y de las coberturas de vacunación, entre otros aspectos, que serán un desafío para el futuro. ${ }^{38,39}$

Los niños son susceptibles a los efectos posteriores de la COVID-19, incluido el aislamiento social y, en muchos casos, la interrupción de la educación. La inclusión de las vacunas COVID 19 en pediatría, con base en las evidencias científicas que vayan surgiendo, permitirá prevenir esta enfermedad, en especial en los pacientes con enfermedades subyacentes, y mitigar los efectos posteriores. ${ }^{40}$

\section{CONCLUSIONES}

La infección por el SARS-CoV-2 en pediatría ocasiona, en la gran mayoría de los casos, un cuadro leve o asintomático. Más de la mitad de los casos refirieron antecedente de contacto con personas con COVID-19 en el entorno familiar. La hospitalización no respondió a criterios clínicos de gravedad. La residencia en barrios vulnerables se comportó como un factor de protección frente a formas graves. La gravedad de la COVID-19 se asoció a la existencia de ciertas comorbilidades y edad menor de 6 meses.

\section{REFERENCIAS}

1. Organización Mundial de la Salud. Información básica sobre la COVID-19. [Acceso: 28 de junio 2021]. Disponible en: https: / / www.who.int/es / news-room/q-a-detail / coronavirus-disease-covid-19

2. Organización Panamericana de la Salud. Actualización EpidemiológicaEnfermedad porcoronavirus(COVID-19)14 de abril de 2021. [Acceso:15 demayo de 2021]. Disponibleen: https: / / www.paho.org/es / documentos/actualizacionepidemiologica-enfermedad-por-coronavirus-covid-1914-abril-2021

3. Mehta NS, MyttonOT, Mullins EWS, Fowler TA, et al.SARSCoV-2 (COVID-19): What Do We Know About Children? A Systematic Review. Clin Infect Dis. 2020; 71(9):2469-79.

4. Viner RM, Mytton OT, Bonell C, Melendez-Torres GJ, et al. Susceptibility to SARS-CoV-2 infection among children and adolescents compared with adults: a systematic review and meta-analysis. JAMA Pediatr. 2021; 175(2):143-56.

5. Alsohime F, Temsah MH, Al-Nemri AM, Somily AM, Al-Subaie S. COVID-19 infection prevalence in pediatric population: Etiology, clinical presentation, and outcome. J Infect Public Health. 2020; 13(12):1791-6.

6. Argentina. Ministerio de Salud. Sala de Situación 
COVID-2019Nuevo Coronavirus2019 Niñez/Adolescencia y COVID-19 Información disponible al 22/03/2021 SE 11 completa. [Acceso: 21 de abril de 2021]. Disponible en: https: / / www.argentina.gob.ar/coronavirus/informesdiarios/sala-de-situacion/informes-especiales

7. Argentina. Ministerio de Salud. Definición de caso de COVID-19. [Acceso: 5 de junio de 2021]. Disponible en: https: / / www.argentina.gob.ar/salud / coronavirus / definicion-de-caso

8. Centers for Disease Control and Prevention. Multisystem Inflammatory Syndrome in Children (MIS-C) Associated with Coronavirus Disease 2019 (COVID-19). Health Alert Network, 14 de Mayo 2020. [Acceso: 15 de mayo de 2021]. Disponible en: https://emergency.cdc.gov/han/2020/ han00432.asp

9. Riphagen S, Gomez X, Gonzalez-Martinez C, Wilkinson N, Thercharis P. Hyperinflammatory shock in children during COVID-19 pandemic. Lancet. 2020; 395(10237):1607-8.

10. Verdoni L, Mazza A, Gervasoni A, Martelli L, et al. An outbreak of severe Kawasaki-like disease at the Italian epicentre of the SARS-CoV-2 epidemic: an observational cohort study. Lancet. 2020; 395(10239):1771-8.

11. Raiden S, Cairoli H, Potasnik J, Di Lalla S, et al. Children hospitalized for COVID-19 during the first winter of the pandemic in Buenos Aires, Argentina. medRxiv. 2020.11.05.20225300.

12. Vasquez L, Sampor C, Villanueva G, Maradiegue E, et al. Early impact of the COVID-19 pandemic on paediatric cancer care in Latin America. Lancet Oncol. 2020; 21(6):7535.

13. Dong $\mathrm{Y}, \mathrm{Mo} \mathrm{X}, \mathrm{Hu} \mathrm{Y}$, Qi X, et al. Epidemiology of COVID-19 Among Children in China. Pediatrics. 2020; 145(6):e20200702.

14. SteinmanJB, Lum JM, Pui-Kay Ho P, KaminskiN, Steinman L. Reduced development of COVID-19 in children reveals molecular checkpoints gating pathogenesis illuminating potential therapeutics. Proc Natl Acad Sci U S A. 2020; 117(40):24620-6.

15. Argentina. Ministerio de Salud. Actualización epidemiológica en población pediátrica de Argentina 14 de julio 2020. [Acceso: 15 de mayo de 2021]. Disponible en: https: / / www.argentina.gob.ar/sites / default / files / actualizacion_epidemiologica_en_poblacion_ pediatrica_14_julio.p $\bar{d} f$

16. Argentina. Ministerio de Salud. Vigilancia, diagnóstico y manejo institucional de casos en pediatría. [Acceso: 22 de mayo de 2021]. Disponible en: https:/ / www.argentina. gob.ar/salud/coronavirus/ casos-pediatria

17. Cao Q, Chen YC, Chen CL, Chiu CH. SARS-CoV-2 infection in children: Transmission dynamics and clinical characteristics. J Formos Med Assoc. 2020; 119(3):670-3.

18. Petrilli CM, Jones S A, Yang J, Rajagopalan H, et al. Factors associated with hospital admission and critical illness among 5279 people with coronavirus disease 2019 in New York City: prospective cohort study. BMJ.2020;369:m1966.

19. Saldías Peñafiel F, Peñaloza Tapia A, Farías Nesvadba D, Farcas Oksenberg K, et al. Manifestaciones clínicas y predictores de gravedad en pacientes adultos con infección respiratoria aguda por coronavirus SARS-CoV-2. Rev Méd Chile. 2020; 148(10):1387-97.

20. Chan JF, Yuan S, Kok KH, To KK, et al. A familial cluster of pneumonia associated with the 2019 novel coronavirus indicating person-to-person transmission: a study of a family cluster. Lancet. 2020; 395(10223):514-23.

21. Figar S, Pagotto V, Luna L, Salto J, et al. Community-level SARS-CoV-2SeroprevalenceSurvey in urban slum dwellers of Buenos Aires City, Argentina: a participatory research. medRxiv. 2020.07.14.20153858.

22. Patel NA. Pediatric COVID-19: Systematic review of the literature. Am J Otolaryngol. 2020; 41(5):102573.
23. Böger B, Fachi MM, Vilhena RO, Cobre AF, et al. Systematic review with meta-analysis of the accuracy of diagnostic tests for COVID-19. Am J Infect Control. 2021; 49(1):21-9.

24. Antony SJ, Almaghlouth NK, Heydemann EL. Are coinfections with COVID-19 and influenza low or underreported? An observational study examining current published literature including three new unpublished cases. J Med Virol. 2020; 92(11):2489-97.

25. Posfay-Barbe KM, Wagner N, Gauthey M, Moussaoui D, et al. COVID-19 in children and the dynamics of infection in families. Pediatrics. 2020; 146(2):e20201576.

26. Castagnoli R, Votto M, Licari A, Brambilla I, et al. Severe acute respiratory syndrome coronavirus 2 (SARS-CoV-2) infection in children and adolescents: a systematic review. JAMA Pediatr. 2020; 174(9):882-9.

27. Liao J, Fan S, Chen J, Wu J et al. Epidemiological and clinical characteristics of COVID-19 in adolescents and young adults. Innovation (N Y). 2020; 1(1):100001.

28. Al Yazidi LS, Al Hinai Z, Al Waili B, Al Hashami H, et al. Epidemiology, characteristics and outcome of children hospitalized with COVID-19 in Oman: A multicenter cohort study. Int J Infect Dis. 2021; 104:655-60.

29. Hong H, Wang Y, Chung HT, Chen CJ. Clinical characteristics of novel coronavirus disease 2019 (COVID-19) in newborns, infants and children. Pediatr Neonatol. 2020; 61(2):131-2.

30. Xia W, Shao J, Guo Y, Peng X, et al. Clinical and CT features in pediatric patients with COVID-19 infection: Different points from adults. Pediatr Pulmonol. 2020; 55(5):1169-74.

31. Rearte A, Baldani A, Barcena Barbeira P, Domínguez C, et al. Características epidemiológicas de los primeros 116.974 casos de COVID-19 en Argentina, 2020. Rev Argent Salud Publica. 2020; 12 (Supl COVID-19):e5.

32. Center for Disease Control and Prevention. Demographic trends of COVID-19 Cases and Deaths in the United States reported to CDC. [Acceso: 8 de junio de 2021]. Disponible en: https://covid.cdc.gov/covid-datatracker/\#demographics

33. BellinoS, Punzo O, Rota MC, Del Manso M, etal. COVID-19 Disease Severity Risk Factors for Pediatric Patients in Italy. Pediatrics. 2020; 146(4):e2020009399.

34. Derespina KR, Kaushik S, Plichta A, Conway EE Jr, et al. Clinical Manifestations and Outcomes of Critically Ill Children and Adolescents with Coronavirus Disease 2019 in New York City. J Pediatr. 2020; 226:55-63.e2.

35. Guan W, NiZ, Hu Y, Liang WH, etal. Clinical Characteristics of Coronavirus Disease 2019 in China. N Engl J Med. 2020; 382(18):1708-20.

36. Argentina. Ministerio de Salud, Gobierno de la Ciudad Autónoma de Buenos Aires. COVID-19 en datos. Situación epidemiológica. [Acceso: 30 de junio de 2021]. Disponible en:https: / / www.buenosaires.gob.ar/coronavirus / datos / situacion-epidemiologica

37. Say D, Crawford N, McNab S, Wurzel D, et al. Postacute COVID-19 outcomes in children with mild and asymptomatic disease. Lancet Child Adolesc Health. 2021; 5(6):e22-3.

38. United Nations. Committee for the Coordination of Statistical Activities (CCSA). How COVID-19 is changing the world: a statistical perspective VolumeII. 2020. [Acceso: 4 de julio de 2021]. Disponible en: https://data.unicef. org/resources / how-covid-19-is-changing-the-world-astatistical-perspective-volume-2/

39. Chiesa V, Antony G, Wismar M, Rechel B. COVID-19 pandemic: health impact of staying at home, social distancing and 'lockdown' measures-a systematic review of systematic reviews. J Public Health (Oxf). 2021:fdab102.

40. Kamidani S, Rostad CA, Anderson EJ. COVID-19 vaccine development: a pediatric perspective. Curr Opin Pediatr. 2021; 33(1):144-51. 